Antonie van Leeuwenhoek 54: 453-463 (1988)

(c) Kluwer Academic Publishers, Dordrecht - Printed in the Netherlands

\title{
BACTERIAL HEMOLYSINS AS VIRULENCE FACTORS
}

Goebel, W., T. Chakraborty and J. Kreft

Institute for Genetics and Microbiology, University of Würzburg, Würzburg, W. -Germany

\section{INTRODUCTION}

Hemolysins are extracellular toxic proteins which are produced by many gram-negative (e.g. E. coli, Serratia spp., Proteus spp., Vibrio spp., Pasteurella spp., Pseudomonas aeruginosa) and gram-positive bacteria (e.g. Streptococcus spp., Staphylococcus aureus, Listeria spp., Bacillus cereus, Clostridium tetani), all of which possess a certain pathogenic potential. Hemolysins have been therefore always considered as virulence factors although direct experimental evidence for this assumption was either poor or non-existent. Most hemolysins cause lysis of erythrocytes by forming pores of varying diameters in the membrane. Many hemolysins can also attack - probably by a similar mechanism - other mammalian cells. Due to this cytolytic effect, they are also termed cytolysins. I shall discuss the following three bacterial hemolysins which we have studied in the past quite extensively with respect to their genetic, biochemical and pathogenic properties: Alpha-hemoylsin from uropathogenic Escherichia coli, Aerolysin from Aeromonas sobria, and Listeriolysin from Listeria monocytogenes.

The hemolysin-producing E. coli strains often infect extraintestinal sites of the human body and can cause cystitis, pyelonephritis or septicaemia. A. sobria frequently infects the intestinal tract but may also cause septicaemia and meningitis. In general both bacteria can be regarded as predominantly extracellular microorganisms colonizing specific tissue surfaces. L. monocytogenes, on the other hand, is a facultative intracellular bacterium which "invades" macrophages or monocytes and survives and multiplies within these professional phagocytes. Whereas high titers of antibodies against several surface antigens are found in the sera of patients suffering from uropathogenic E. coli or Aeromonas sobria (hydrophila) infections only low titers of antibodies to L. monocytogenes are normally detectable in patients with an acute listeriosis, and T cell-immunity is considered the major protective host response against a $L$. monocytogenes infection ( 1 ).

In the following mainly the role of the three hemolysins as virulence factors will be discussed but one should keep in mind that other bacterial components such as adhesins, serum resistance, capsules, iron transport systems and other virulence factors are connected with the pathogenicity of these bacteria (23). 
RESULTS AND DISCUSSION

A.) The genetic determinants of hemolysin formation in E. coli, Aeromonas sobria and Listeria monocytogenes and their regulation

a) $\alpha$-Hemolysin of E. coli

As shown by our group (2); the genetic determinant for $\alpha$-hemolysin of $E$. coli consists of four genes ( $h l y C, A, B, D$ ) which were sequenced and the amino acid composition of the expected four gene products were deduced from this DNA sequence $(3,4)$. It was further shown $(5)$ that the hly-determinant can be located either on transmissible plasmios (5) or on specific large inserts in the chromosome of $E$. coli (6). High homology exists within the four structural hly genes between several chromsome and plasmid-encoded E. coli hly determinants (7). Interestingly, high sequence homology with the E. coli hly determinant has been recently demonstrated also for the hly determinants of Proteus mirabilis, P. vulgaris, and Morganella morganii $(8,9)$,

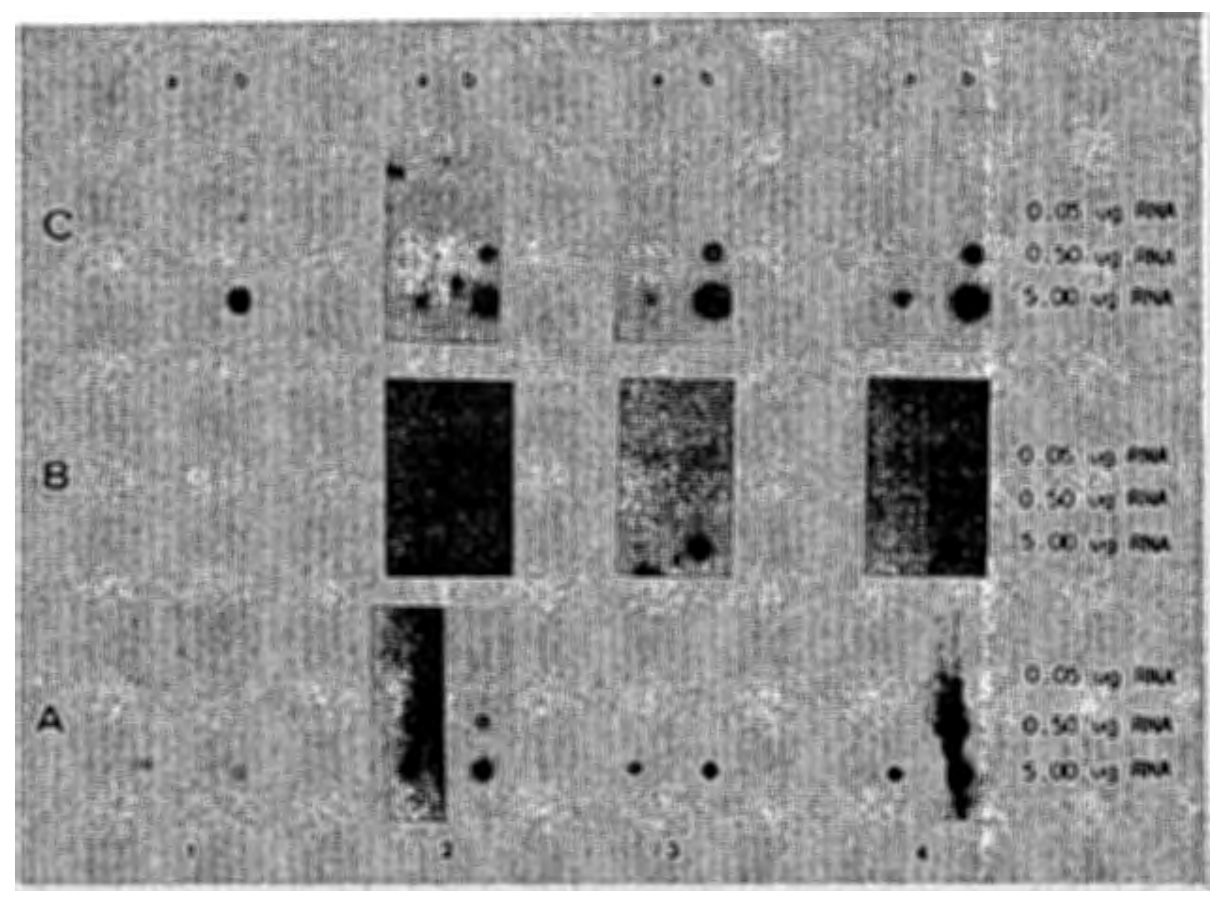

Fig. 1 The influence of hlyR on the transcription of hlyC, $A, B$ and $D$. Dot blots with increasing agmounts of total cellular RNA $(0.05 \mathrm{ug}$, $0.5 \mathrm{ug}$ and 5 ug RNA) and ${ }^{2}$-labeled specific gene probes were performed. (A) RNA hybridized with a hlyC, A - specific probe, (B) RNA hybridized with a hlyB - specific probe and (C) RNA hybridized with a hlyD - specific probe. The RNA in positions $1_{8} 2,3$ and $\&$ were taken at cell densities of $2 \times 10^{8}, 4 \times 10^{8}, 7 \times 10^{8}$ and $10^{\circ} \mathrm{cells} / \mathrm{ml}$, respectively. (a) marks hybridization with RNA from E. coli $5 K$ carrying pANN202-312 ( $\mathrm{hly} \mathrm{R}^{-}$) and (b) from E. coli 5K carrying pANN202$812\left(\right.$ hlyR ${ }^{+}$). 
and the genetic determinant for leukotoxin (ltx) of Pasteurella haemolytica (10). On the contrary, little sequence homology was observed between several E.coli determinants in the upstream and downstream regions flanking the four structural hly genes. A region (upstream of hlyc) of more than $400 \mathrm{bp}$ was defined as a regulatory site in a chromosomal hly determinant which apparently contain two promoters from which a polycistronic $m-R N A$ is transcribed (11). A terminator-like structure was observed in all hly determinants in the intercistronic region between hlyA and hlyB and evidence was presented that most transcripts terminate at this point (11). In a plasmidencoded hly determinant, we have recently identified (12) a cis-acting element, which is located almost $2 \mathrm{~kb}$ upstream from the start of hlyC. The region between $\mathrm{hlyC}$ and this element, termed hlyR, includes two promoters and an IS element 467 bp from the start of hlyC. HlyR enhances transcription of hlyC and hlyA two to threefold, and that of hlyB and hlyD at least 50 fold (Fig. 1) and leads to 50 to 100 fold stimulation of secreted hemolysin compared to the hlyR-negative situation (12). Whether hlyR leads to an antitermination of transcription at the hlyA terminator and hence to the enhanced transcription of $\mathrm{hlyB}$ and $\mathrm{hlyD}$ or whether hlyR enhances transcription of hlyB and hlyD directly from specific promoters can not be decided at the moment, but preliminary data argue in favor of the second assumption (Hess and Gentschev, unpublished results).

b) Aerolysin of Aeromonas sobria

The aerolysin gene from a clinical isolate Aeromonas strains AB3 serotype has been cloned and its entire nucleotide sequence determined. The gene encodes a preproprotein of 492 amino acids with a molecular weight of $54.4 \mathrm{kD}$. Genetic mapping of the aerolysin gene (aer $A$ ) has shown that flanking regions both upstream (aerC) and downstream (aerB) of the gene affect its synthesis. Recently, we have determined the promoter for the aerolysin gene and have found it to be one of two non-overlapping divergent promoters present within the aerC region $5^{\circ}$ of the aerolysin gene. The aerC region is characterised by its extreme $A+T$ content ( $68 \%$ as compared to $41 \%$ for the structural gene), eight copies of a core motif aATAAAa, and its absence of any extended open reading frames for $340 \mathrm{bp}$. The second promoter transcribes a hitherto unidentified $6,4 \mathrm{kD}$ polypeptide whose role in toxin regulation is as yet undetermined. The downstream aer $B$ region encodes a $82 \mathrm{kD}$ protein transcribed in the same orientation as aer $A$ from an independent promoter. The functional role of aerB in aerolysin regulation is at present unclear. However, A. sobria mutants carrying deletions within this region have altered surface properties and grow poorly in iron-restricted media.

$A$ comparison of the aerolysin. sequence determined from a $A$. hydrophila fish isolate to that of the $A$. sobria aerolysin shows $\frac{A}{a n}$ overall homology of $77 \%$ at both nucleotide and amino acid levels. However, the corresponding aerC region for the two isolates showed only a $46 \%$ homology at the nucleotide level, despite identity in their $(A+T)$ content. In a study using 300 strains from diverse geographical regions the aerolysin gene was found to be present in 87 $\%$ of all hemolytic. Aeromonas isolates irregardless of whether they 
were from clinical or environmental sources while the aerolysin gene was present as a single copy in many strains, isolates were found that carried two copies of the aerolysin gene (Husslein and Chakraborty, unpublished results).

c) Listeriolysin of $L$. monocytogenes

The gene for listeriolysin (IisA) has been cloned recently from two

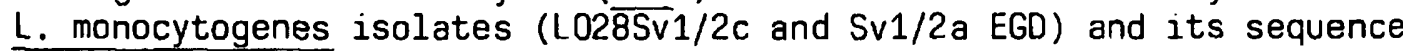
determined (15, Leimeister-Wächter and Chakraborty, unpublished data). An almost full-length lisA gene probe hybridizes in a single band with the chromosomal DNAs of Listeria monocytogenes, $L$. ivanovii and $L$. seeligeri (Leimeister-Wächter, Chakraborty, Haas, unpublished results) indicating that considerable sequence homologies exist between the genes encoding the hemolysins from these species. This finding is in contrast to our previous observation (16) and those of others (15) which failed to detect homology with the $L$. ivanovii and $L$. seeligeri genes using shorter lisA gene probes and suggests that homology

Mly dotorminants from:

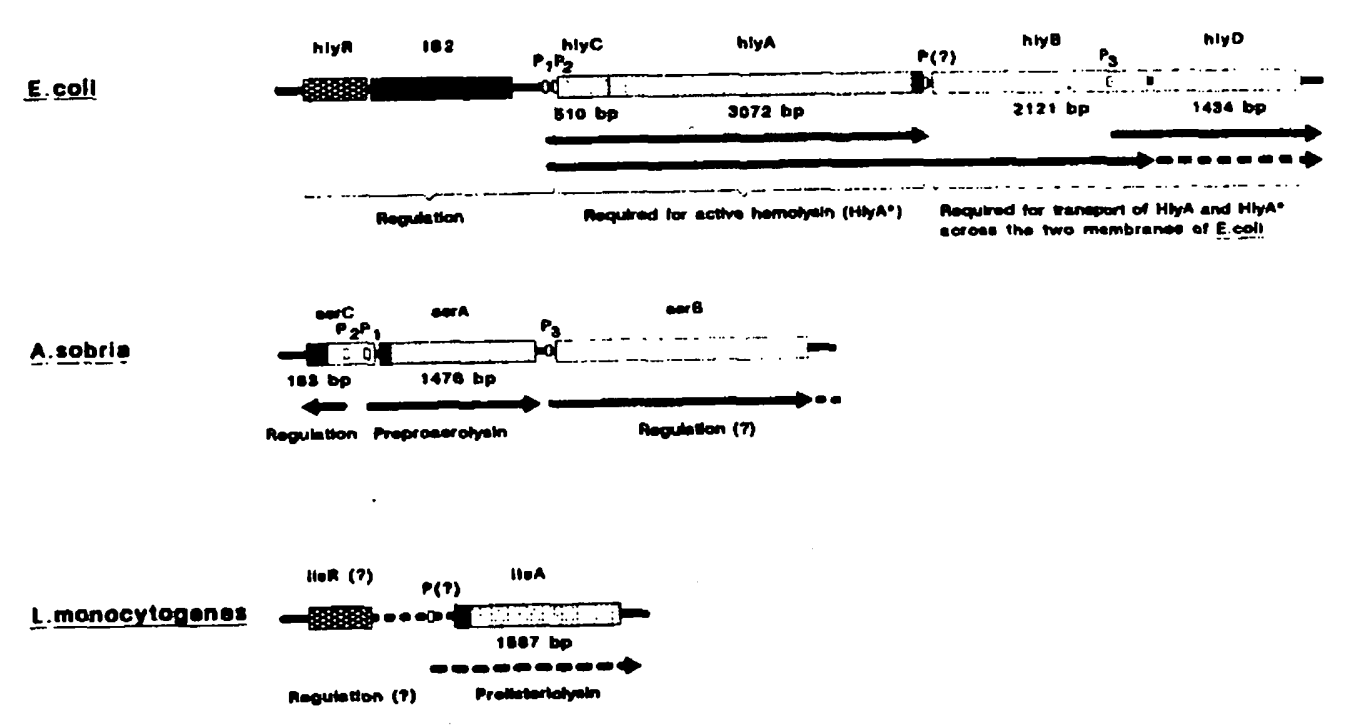

Fig. 2 The genetic determinants for $\alpha$-hemolysins of $E$. col $i$, aerolysin of Aeromonas sobria and listeriolysin of Listeria monocytogenes (see text for details) 
between the three listeriolysin genes may be limited to only part of the gene. In addition, the lisA gene shows homology to a C-terminal region (containing the single cys codon) of two other SH-activated cytolysins (Streptolysin 0 and Pneumolysin). A functional promoter for lisA has not yet been defined. Recent data (Sokolovic and Goebel, manuscript in preparation) indicate that lisA is expressed under heat shock conditions together with 12 to 14 other heat-shock proteins. Listeriolysin-negative mutants were obtained by transposon-mutagenesis. with In916 $(17,18)$. Whereas one class of these Hly mutants contains the insertion within the structural lisA gene and expresses a truncated LisA protein $(17,18)$, the other class (17) produces very low or undetectable levels of listeriolysin, suggesting a defect in lisA gene expression. One subclass of the latter mutants appears to be impaired in the expression of most other heat shock proteins. The transposon insertion of one $\mathrm{hly}^{-}$mutant belonging to this subclass was shown to be located upstream of the start of lisA. Fig. 2 summarizes the essential features of the three hemolysin determinants and their regulation.

B.) Synthesis and Secretion of the Hemolysins from E. coli, A. sobria and $L$. monocytogenes

Alpha-hemolysin of $E$. coli is synthesized and secreted in the logarithmic growth phase. Hemolytic activity in the supernatant correlates with the secretion of a $110 \mathrm{kD}$ protein, the amino acid composition of which corresponds to the umprocessed hlyA gene product. In the late log phase and in the stationary phase hemolytic activity in the supernatant drops to a low level in spite of the presence of undegraded $\mathrm{HlyA}$ protein in the supernatant. The hlyC gene product is required for rendering $\mathrm{Hl} y \mathrm{~A}$ hemolytically active $(5,19)$. The mechanism of this activation is still unknown, but active HIyA ( $\mathrm{HlyA}^{*}$ ) can be distinguished from inactive HlyA protein by several criteria: (a) The isoelectric point of $\mathrm{HIYA}^{\star}$ is $\mathrm{pH} 4.0$ whereas that of $\mathrm{HlyA}$ is $\mathrm{pH} 6.2$ (as expected by the amino acid composition of $\mathrm{HlyA}$ ), (b) $\mathrm{HlyA}^{*}$ Ioses activity quickly upon treatment with phospholipase $C$ and ultrasonication (20), (c) $\mathrm{HlyA}^{\star}$ is more stable against tryptic digestion than $\mathrm{HlyA}$ and (d) $\mathrm{HlyA}^{*}$, but not $\mathrm{HlyA}$, forms pores of $1-2 \mathrm{~nm}$ in the erythrocyte membrane and in artificial lipid bilayers (21). These observations have lead us to speculate that one or more acidic phospholipids may be complexed to HlyA to yield the closed conformation of $\mathrm{HIyA}^{*}$ and this "activation process" may be catalyzed by $\mathrm{HlyC}$.

Using site specific mutagenesis we could identify four functional domains in $\mathrm{HlyA}$, one of which appears to be connected to the activation process. There are three pronounced hydrophobic regions in HlyA between amino acids 270 to 350 . Removal of the internal hydrophobic region (21 amino acids) leads to the loss of hemolytic activity and of pore formation in a lipid bilayer film. The isoelectric point of this mutant $\mathrm{HlyA}$ remains at $\mathrm{pH} 6.2$ even in the presence of $\mathrm{HIYC}$ suggesting that activation by $\mathrm{HIyC}$ may no longer occur.

All toxins related to the $\alpha$-hemolysin of $E$. coli (see above) have in common a repeat domain which consists in the case of HlyA of 11 repeats each of which is 9 amino acids long. In HlyA this repeat domains extends from amino acid position 739 to 849 (21). These 
apparently related toxins have been therefore recently termed RTX (Repeat Toxins) (Welch, RA, personal communication). Removal of a single repeat or combinations of several repeats leads to the loss of hemolytic activity in the presence of low $\mathrm{Ca}^{2+}$ concentration (21). Hemolytic activity of some of these repeat deletion mutants can be restored by elevated concentrations of $\mathrm{Ca}^{2+}$. The ability to form pores in artificial lipid bilayer films is unaffected in all repeat mutants. This has led us to suggest that the repeat domain of HIyA is the recognition site for a "receptor" on the erythrocyte membrane and that the proper conformation of this repeat region depends on the presence of $\mathrm{Ca}^{2+}(21)$.

The $\mathrm{N}$-terminal end of $\mathrm{H} I$ yA does not carry a typical transport signal but has amphiphilic properties and may therefore interact with membranes (22).

The actual "transport signal" of $\mathrm{HlyA}$ for its export mediated by $\mathrm{HlyB}$ and $\mathrm{HlyD}$, is located on the C-terminal end. Fusion of the last 37 amino acid to a ( $N$-terminal) signal-less alkaline phosphatase gene (phoA) leads to the efficient transport of this fusion protein across both membranes of $\mathrm{E}$. coli in the presence of active $\mathrm{HlyB}$ and $\mathrm{HlyD}$

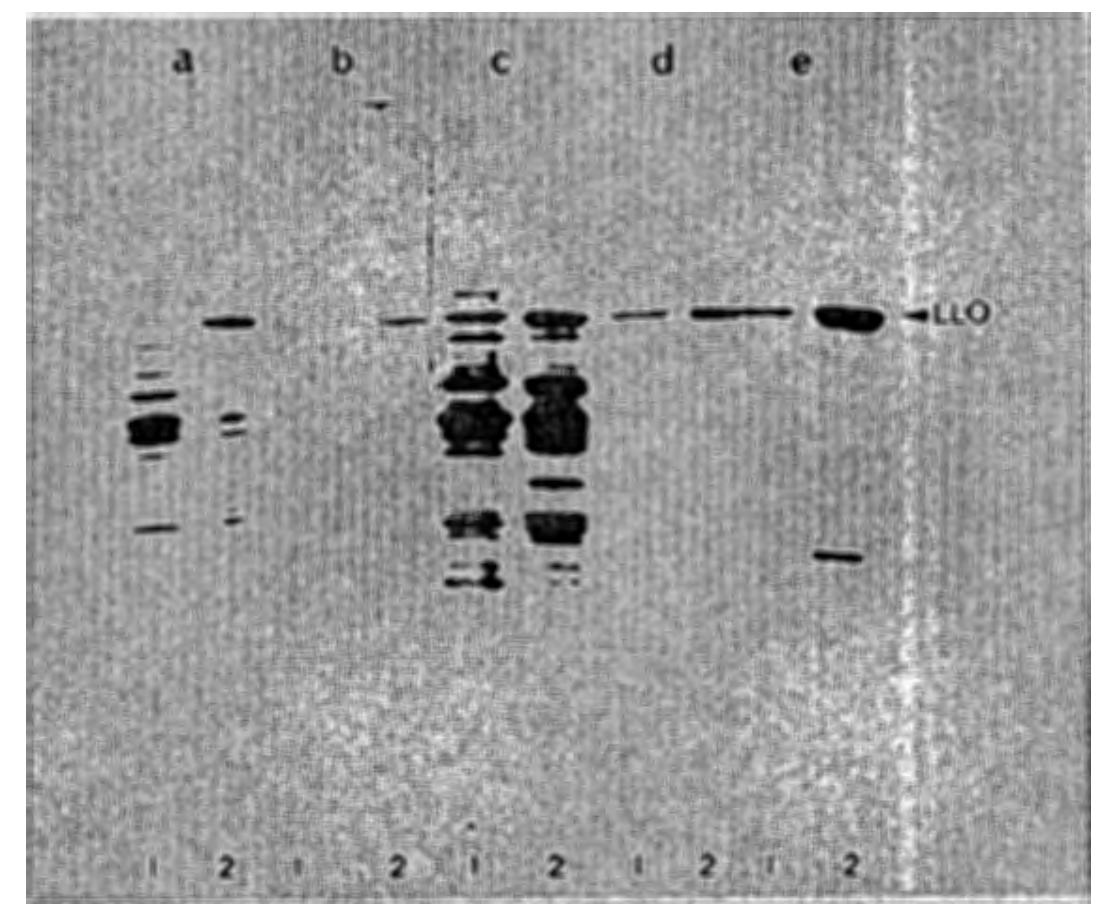

Fig. 3 Cell-associated (a-d) and cell-free listeriolysin (e) synthesized at $37^{\circ} \mathrm{C}(a, b)$ and $48^{\circ} \mathrm{C}(\mathrm{c}-\mathrm{e})$ in the two $\mathrm{L}$. monocytogenes strains (1) Sv1/2a EGD (termed "EGD strain" provided by S. Kaufmann, Ulm, Germany) and (2) Sv1/2a SLCC5764 (termed "Mackaness strain"). (a) and (c) show the proteins labeled at $37^{\circ} \mathrm{C}$ and $48^{\circ} \mathrm{C}$, respectively, (b) and (d) șow immunoblots of the same protein patterns developed with anti-SLO antibodies which specifically react with listeriolysin (LLO), and $(e)$ shows the external listeriolysin synthesized and secreted at $48^{\circ} \mathrm{C}$. 
(Hess J, Jarchau $T$ and Goebel $W$, unpublished results). The precise function of the two transport proteins for $\mathrm{HlyA}, \mathrm{HlyB}$ and $\mathrm{HI} \mathrm{yD}$, both of which are incorporated in the $E$. coli membrane, is still unknown. $B t$ the $\mathrm{N}$-terminal end (removal of a 23 amino acid transport leader sequence) and the C-terminal end (removal of the last 27 amino acids). Whereas cleavage of the $\mathrm{N}$-terminal signal sequence occurs during transport of aerolysin across the cytoplasmic membrane, cleavage at the C-terminal end appears to occur outside the cell. This cleavage is necessary for hemolytic activity but may not be directly connected with the transport of aerolysin. However, transport of the aerolysin protein which lacks the processed $\mathrm{Ct}$ the $\mathrm{N}$-terminal end (removal of a 23 amino acid transport leader sequence) and the $C$-terminal end (removal of the last 27 amino acids). Whereas cleavage of the $\mathrm{N}$ terminal signal sequence occurs during transport of aerolysin across the cytoplasmic membrane, cleavage at the C-terminal end appears to occur outside the cell. This cleavage is necessary for hemolytic activity but may not be directly connected with the transport of aerolysin. However, transport of the aerolysin protein which lacks the processed C-terminal end due to a deletion in the aerA gene is considerably hampered in Aeromonas and in E. coli. This mutant aerolysin appears to quickly aggregate, suggesting that the transport competent conformation of aerolysin may require the $\mathrm{C}$-terminal end. There is evidence that a short amino acid sequence of about 20 aa which immediately follows the transport signal in AerA is needed for the efficient transport of aerolysin across the outer membrane (Huhle and Chakraborty, unpublished results).

Listeriolysin seems to be produced in a precursor form containing a putative transport signal sequence of 25 amino acids (15) which is apparently cleaved off during transport across the Lister ial membrane. Most of the listeriolysin protein $(58 \mathrm{kD})$ is found in the supernatant but a substantial portion remains cell-associated at the normal growth temperature and under heat-shock conditions (Fig. 3). Unlike hemolysin of $E$. coli and aerolysin, listeriolysin does not appear to require special activation or specific proteolytic processing to gain hemolytic activity.

C. In vitro and in vivo actions of the hemolysins

E. coli $\alpha$-hemolysin. The involvement of hemolysin in virulence was demonstrated in rat, mouse and chicken embryo (23) using genetically well defined $\mathrm{Hly}^{-}$deletion mutants and reconstituted $E$. coli strains into which different hly determinants and genetically altered hly genes were introduced $\overline{(24)}$. It was further shown (25) that not only the amount of hemolysin correlates with the degree of toxicity, but that structural features of HlyA may also influence the virulence property. Furthermore, purified hemolysin stimulates arachidonic acid metabolism in granulocytes and causes release of the lipid mediators such as leukotrienes. Whether this effect is caused by $\mathrm{Ca}^{2+}$ influx into the pores formed by hemolysin or whether hemolysin triggers the intracellular event in a sublytic dose remains to be answered. Cytotoxic damage of mouse fibroblasts and human leucocytes by hemolysin was observed, whereas human lymphocytes were relatively insensitive to this toxin (27). 
Aerolysin. The aerolysin gene aerA was deleted from the chromosome of A. sobria by marker exchange mutagenesis (28). The thus obtained $\mathrm{Hly}^{-}$ mutant $A B 3-25$ exhibited a significantly reduced toxicity in the mouse (28) when the mutant strain was infected intraperitoneally. No effect on toxicity was observed with a mutant in which aerB was partially deleted. A deletion mutant AB3-5 in which parts of $\overline{\operatorname{aer} A}$ and aerB were deleted by marker exchange exhibited the same reduced toxicity as AB325. Strong demonecrotic lesions with the formation of large zones of necrosis occured when the wild-type strain $A B 3$ was injected subcutaneously into healthy mice. Surviving animals contain high titers of antibodies agains aerolysin in the blood. The mutant AB3-5, in contrast, developed only small lesions which healed within a few days. In this subcutaneous infection model the mortality rate was high when mice were infected with the wild-type strain $A B 3$, but low when infected with the mutant strain AB3-5.

Listeriolysin. Virulent strains of $L$. monocytogenes are hemolytic due to the production of listeriolysin. Listeriolysin has therefore long been considered as an essential virulence factor of this facultative intracellular bacterial pathogen. On the other hand, $L$. ivanovii and $L$. seeligeri are also hemolytic and, as pointed out before, the genes for these listeriolysins exhibit homology with the lisA gene of $L$. monocytogenes. Yet, $L$. seeligeri is avirulent and $L$. ivanovii is only slightly virulent for humans (29). There is also a lack of correlation between the levels of cytolytic in vitro activity at normal growth temperatures and virulence of $L$. monocytogenes strains (30). However, the recent finding that listeriolysin is induced in $\mathrm{L}$. monocytogenes strains under heat-shock conditions suggests that this toxin is synthesized more efficiently under stress conditions, which the intracellular environment, especially the phagosomal milieu, may impose on invading bacteria. In addition, the isolation of transposoninduced mutants from L. monocytogenes which fail to synthesize active hemolysin $(17,18)$ or do so at very low levels $(17)$ has unambiguously demonstrated the involvement of listeriolysin in the pathogenesis of $L$. monocytogenes. Both types of listeriolysin-negative mutants are avirulent when tested in mice. Moreover, these mutants are unable to survive in mouse peritoneal macrophages and in non-professional phagocytic cells, although the mutant bacteria can still enter these mammalian host cells. Invasion of the non-professional phagocytes is not observed by the avirulent Listeria species, even by the hemolytic ones such as L. seeligeri or L. ivanovii (31). The invasion step probably requires, among other yet unknown components of $L$. monocytogenes, an extracellular protein $(p 60)$, which is absent or altered in the non-invasive Listeria species (32). Evidence was presented (33) that listeriolysin may disrupt the phagosomal membrane, thereby allowing the bacterial cells to escape into the cytoplasm before lysosomes can fuse to the phagosome or afterwards. Whether this is the actual and only in vivo function of listeriolysin remains to be investigated. 


\section{ACKNOWLEDGEMENTS}

The authors are indebted to $M$. Leimeister-Wächter, J. Hess, I. Gentschev, A. Haas, Z. Sokolovic, B. Huhle, A. Ludwig, M. Kuhn, V. Husslein, F. Garcia, I. Thautphoeus for allowing us to quote their unpublished results. We also wish to thank $E$. Appel for editorial assistance and $M$. Wuenscher for critical reading of the manuscript. This work was supportd by grants from the Deutsche Forschungsgemeinschaft (SFB 165 - B4, SFB 176 - B8).

\section{REFERENCES}

1. Mackaness GB: Cellular resistance to infections. J. Exp. Med. 116, 381-406 (1962)

2. Wagner $W$, Vogel $M$ and Goebel $W$ : Transport of hemolysin across the outer membrane of $E$. coli requires two functions. J. Bact. $154,200-210$ (1983)

3. Hess $J$, Wels $W$, Vogel $M$ and Goebel $W$ : Nucleotide sequence of a plasmid-encoded hemolysin determinant and its comparison with a corresponding chromosomal hemolysin sequence. FEMS Microbiol. Letts. 34, 1-11 (1986)

4. Felmlee $T$, Pellet $S$ and Welch RA: Nucleotide sequence of an Escherichia coli chromosomal hemolysin. J. Bact. 163, 94-105 (1985)

5. Noegel $A$, Rdest $U$ and Goebel $W$ : Determination of the functions of hemolytic plasmid pHly152 of Escherichia coli. J. Bact. 145, 233-247 (1981)

6. Knapp S, Hacker J, Jarchau T and Goebel W: Large, unstable inserts in the chromosome affect virulence properties of uropathogenic E. coli 06 strain 536. J. Bact. 168, 22-30 (1986)

7. Müller G, Hughes $C$ and Goebel W: Relationship between plasmid and chromosomal hemolysin determinants of $E$. coli. J. Bact. $153,846-851$ (1983)

8. Koronakis V, Cross M, Senor B, Koronakis E and Hughes C: The secreted hemolysins of Proteins mirabilis, Proteus vulgaris and Morganella morganii are genetically related to each other and to the alpha-hemolysin of E. coli. J. Bact. 169, 1509-1515 (1987)

9. Welch RA: Identification of two different hemolysin determinants in uropathogenic Proteus isolates. Infect. Immun. $55,2183-2190$ (1987)

10. Strathdee CA and Lo, RYC: Extensive homology between the leukotoxin of Pasteurella haemolytica $A 1$ and the alphahemolysin of $E$. coli. Infect. Immun. 55, 3233-3236 (1987)

11. Welch RA and Pellett S: Transcriptional organization of the $E$. coli hemolysin genes. J. Bact. 1622-1630 (1988)

12. Vogel M, Hess J, Then I, Juarez $A$ and Goebel W: Characterization of a sequence (hlyR) which enhances synthesis and secretion of hemolysin in E. coli. Mol. Gen. Genet. 212, 76-84 (1988)

13. Chakraborty $T$, Huhle $B, B e r g b a u e r ~ H$ and Goebel $W$ : Cloning, expression, and mapping of the Aeromonas hydrophila aerolysin gene determinant in E. coli K-12. J. Bact. 167, 368-374 (1986) 
14. Husslein V, Huhle B, Jarchau T, Lurz R, Goebel W and Chakraborty $T$ : Nucleotide sequence and transcriptional analysis of the aerC aerA region of Aeromonas sobria encoding aerolysin and its regulatory region. Molec. Microbiol. 2, (in press)

15. Mengaud J, Vicente M-F, Chenevert J, Peiveira JM, Geoffrey C, Gicquel-Sanzey B, Baquereo F, Perez-Diaz, J-C and Cossart P:

Expression in $E$. coli and sequence analysis of the listeriolysin 0 determinant of Listeria monocytogenes. Infect. Immun. 56, 766-772 (1988)

16. Goebel W, Kathariou S, Kuhn M, Sokolovic Z, Kreft J, Köhler S, Funke $D$, Chakraborty $T$ and Leimeister-Wächter $M$ : Hemolysin from Listeria - biochemistry, genetics and function in pathogenesis. Infection 16, 149-156 (1988)

17. Kathariou S, Metz P, Hof $H$ and Goebel W: Tn916-induced mutations in the hemolysin determinants affecting virulence of Listeria monocytogenes. J. Bact. 169, 1291-1297 (1987)

18. Gaillard JL, Berche $P$ and Sansonetti P: Transposon mutagenesis as a tool to study the role of haemolysins in virulence of Listeria monocytogenes. Infect. Immun. 52, 50-55 (1986)

19. Härtlein M, Schießl S, Wagner $W$, Rdest U, Kreft $J$ and Goebel W: Transport of hemolysin by $E$. coli. J. Cell. Biochem. 22, 87-97 (1983)

20. Wagner $W$, Kuhn $M$ and Goebel $W$ : Active and inactive forms of hemolysin ( $\mathrm{HlyA}$ ) from E. coli. Biol. Chem. Hoppe-Seyler 369, $39-46$ (1988)

21. Ludwig $A$, Jarchau $T$, Benz $R$ and Goebel $W$ : The repeat domain of E. coli haemolysin (HLyA) is responsible for its $\mathrm{Ca}^{2+}$-dependent binding to erythrocytes. Mol. Gen. Genet. (in press)

22. Erb K, Vogel M, Wagner $W$ and Goebel $W$ : Alkaline phosphatase which lacks its own signal sequence becomes enzymatically active when fused to $N$-terminal sequences of $E$. coli haemolysin ( $\mathrm{H} l y A)$. Mol. Gen. Genet. 208, 88-93 (1987)

23. Hacker $J$, Hof $H$, Emödy $L$ and Goebel $W$ : Influence of cloned $E$. coli hemolysin genes, $S$-fimbriae and serum resistance on pathogenicity in different animal models. Microbial. Pathogen. $1,533-547$ (1986)

24. Hacker J, Hof H, Emödy L and Goebel W: Clones hemolysin genes from $E$. coli that cause urinary tract infection determine different levels of toxicity in mice. Infect. Immun. 42, 57-63 (1983)

25. Goebel W, Kathariou S, Hacker W, Chakraborty T, LeimeisterWächter $M$, Ludwig $A$, Hess $J$, Kuhn $M$ and Wagner $W$ : Bacterial cytolysins as virulence factors, in Fehrenbach et al. (Eds.) Bacterial Protein Toxins, Zbl. Bakt. Suppl. 325-335, GustavFischer, Stuttgart, New York, 1988

26. König B, König W, Scheffer J, Hacker J and Goebel W: Role of $E$. coli hemolysin and bacterial adherence in infection: requirement for release of inflammatory mediates from granulocytes and mast cells. Infect. immun. 54, 886-899 (1986)

27. Gadeberg $\mathrm{OV}$ and Orskov J: In vitro cytotoxic effect of hemolytic E. coli on human blood granulocytes. Immun. 45, 255261 (1984) 
28. Chakraborty $T$, Huhle B, Hof $H$, Bergbauer $H$ and Goebel W: Marker exchange mutagenesis of the aerolysindeterminant in Aeromonas hydrophila demonstrates the role of cereolysin in $A$. hydrophila-associated systemic infections. Infect. Immun. 55, 2274-2280 (1987)

29. Rocourt J, and Seeliger HPR: Is haemolysis an in vitro marker of the pathogenic strains of the genus Listeria. Ann. Microbiol. (Institut Pasteur 138, 277-279 (1987)

30. Kathariou S, Rocourt J, Hof $H$ and Goebel W: Levels of $L$. monocytogenes hemolysin are not directly proportional to virulence in experimental infections of mice. Infect. Immun. $56,534-536$ (1988)

31. Kuhn $M$, Kathariou $S$ and Goebel $W$ : Hemolysin supports survival but not entry of the intracellular bacterium Listeria monocytogenes. Infect. Immun. 56, 79-82 (1988)

32. Kuhn $M$ and Goebel $W$ : Identification of an extracellular protein of $L$. monocytogenes possibly involved in intracellular uptake by mammalian cells. Infect. Immun. (in press)

33. Gaillard JL, Berche P, Mounier J, Richard S and Sansonetti P: In vitro model of penetration and intracellular growth of $L$. monocytogenes in the human enterocyte-like cell line Caco-2. Infect. Immun. 55, 2822-2829 (1987) 